[Article]

\title{
高硅MFI分子筛膜的合成与低温脱除膜内有机模板剂
}

\author{
赵淑蘅 ${ }^{1,2}$ 郎 林 ${ }^{1, *} \quad$ 江俊飞 ${ }^{1,2} \quad$ 阴秀丽 ${ }^{1}$ 吴创之 ${ }^{1}$ \\ ('中国科学院广州能源研究所, 中国科学院可再生能源重点实验室, 广州 510640; ${ }^{2}$ 中国科学院大学, 北京 100049)
}

摘要: 采用旋涂法在粗粘的 $\alpha-\mathrm{Al}_{2} \mathrm{O}_{3}$ 载体片上制备出较完备的分子篎晶种层; 以四丙基氢氧化铵(TPAOH)为 有机模板剂, 通过调控合成液的 $\mathrm{H}_{2} \mathrm{O} / \mathrm{Si}$ 摩尔比, 实现了对分子篎晶体面内优先生长的调控; 经过三次水热合 成得到致密交联的 $h 0 h$-轴取向高硅MFI 分子篎膜, 膜厚约为 $8 \mu \mathrm{m}$ (包括 $5 \mu \mathrm{m}$ 致密层和 $3 \mu \mathrm{m}$ 过渡层)。采用 先低温加氢裂解后低温空气氧化的两步法脱除工艺, 有效脱除了分子篮膜内的有机模板剂。相比于传统高温 制烧法, 该法可以避免分子篮膜因脱除模板剂而形成的较大晶间缺陷。因而采用低温两步法脱除模板剂的分 子篮膜片在 $30^{\circ} \mathrm{C}$ 时具有较好的 $\mathrm{CO}_{2}$ 分离效果, 其 $\mathrm{CO}_{2} / \mathrm{N}_{2}$ 分离因子达到 $5.2, \mathrm{CO}_{2}$ 渗透通量高达 $5.8 \times 10^{-7}$ $\mathrm{mol} \cdot \mathrm{m}^{-2} \cdot \mathrm{s}^{-1} \cdot \mathrm{Pa}^{-1}$ 。

关键词：高硅MFI分子節膜；模板剂脱除；低温加氢裂解；气体膜分离；载体

中图分类号: 0647

\section{Synthesis and Low-Temperature Detemplation of High-Silica MFI Zeolite Membranes}

\author{
ZHAO Shu-Heng ${ }^{1,2} \quad$ LANG Lin ${ }^{1, *} \quad$ JIANG Jun-Fei ${ }^{1,2} \quad$ YIN Xiu-Li ${ }^{1} \quad$ WU Chuang-Zhi ${ }^{1}$ \\ ( ${ }^{1}$ CAS Key Laboratory of Renewable Energy, Guangzhou Institute of Energy Conversion, Chinese Academy of Sciences, \\ Guangzhou 510640, P. R. China; ${ }^{2}$ University of Chinese Academy of Sciences, Beijing 100049, P. R. China)
}

\begin{abstract}
High-silica MFI zeolite membranes supported on porous $\alpha$-alumina discs were prepared by a seeded secondary growth method, using tetrapropylammonium hydroxide (TPAOH) as organic template. First, nanocrystals were deposited on rough $\alpha-\mathrm{Al}_{2} \mathrm{O}_{3}$ discs by a spin-on process. Then, based on controlling the $\mathrm{H}_{2} \mathrm{O}$ / Si molar ratio of the synthetic solution, a restricting in-plane $h 0 h$-oriented growth method with an ultra-dilute precursor was designed to prepare non-defective zeolite membranes that were as thin as possible. Finally, crosslinked and dense MFI zeolite membranes were prepared after the third synthesis step, giving a membrane layer thickness of about $8 \mu \mathrm{m}$, including $\sim 5 \mu \mathrm{m}$ dense layers and $\sim 3 \mu \mathrm{m}$ intermediate layers. A novel, two-step method, coupling by low-temperature hydrocracking and oxidation, is proposed for efficient removal of the template from zeolite membranes. Compared with traditional high-temperature calcination, template removal by the two-step method could eliminate the grain boundary defects formed in response to stresses induced by heat treatment. As a result, the membranes treated by the two-step detemplation method displayed a preferable $\mathrm{CO}_{2} / \mathrm{N}_{2}$ separation factor (about 5.2) and high $\mathrm{CO}_{2}$ permeance $\left(5.8 \times 10^{-7} \mathrm{~mol} \cdot \mathrm{m}^{-2} \cdot \mathrm{s}^{-1} \cdot \mathrm{Pa}^{-1}\right)$ at $30^{\circ} \mathrm{C}$.
\end{abstract}

Key Words: High-silica MFI zeolite membrane; Template removal; Low-temperature hydrocracking; Gas permeation; Support

Received: September 24, 2015; Revised: November 23, 2015; Publishen on Web: November 24, 2015.

${ }^{*}$ Corresponding author. Email: langlin@ms.giec.ac.cn; Tel:+86-20-37218289.

The project was supported by the National Natural Science Foundation of China $(51202245,51106165)$ and Natural Science Foundation of Guangdong Province, China (S2013010014896, 10251007006000000).

国家自然科学基金(51202245, 51106165)和广东省自然科学基金(S2013010014896, 10251007006000000)资助项目

(C) Editorial office of Acta Physico-Chimica Sinica 


\section{1 引言}

分子篎膜是一种由分子篮微晶相互交联生长 而成的新型无机膜材料, 具有规则的孔道结构, 孔径在 $0.3-1.0 \mathrm{~nm}$ 之间, 与一般分子的直径大小 相近, 具有优良的膜分离性能, 被广泛应用于膜 分离和膜催化等诸多领域 ${ }^{1-7}$ 。分子篮膜的种类很 多, 其中 MFI 分子篎膜因其特殊的孔道结构和相 对成熟的合成工艺等特点而受到广泛关注 ${ }^{8-19}$ 。

分子篮膜大多是在多孔载体上合成的复合膜 材料, 因而载体的物理性质 ${ }^{18,20,21}$ (如粗䊁度、孔隙 率等)和化学性质 16,22-27 会显著影响 MFI 分子篮膜的 完备性和分离特性。一般认为, 在较为粗糙的大 孔载体表面直接生长连续且完备的分子篮膜的难 度较大, 大多需要先制备一层较薄的平滑过渡层 或进行表面化学修饰, 进而在改性后的载体上合 成完备的分子篎膜 ${ }^{25,28-31}$; 但其制备工艺相对复 杂, 且成本较高。此外, 如何有效避免在热处理 脱除膜内有机模板剂时形成的缺陷, 也是影响 MFI 分子篮膜制备与应用的一个重要因素。Choi 等 ${ }^{32}$ 的 研究结果表明, 由于膜层与载体的热膨胀系数存 在差异，以及分子篮晶体本身各轴向间的热膨胀 方向和幅度也不同, 所以采用传统高温炦烧工艺 时容易在分子篮膜内产生裂纹等缺陷，进而会显 著影响分子笁膜的分离效果。Dong 等 ${ }^{33}$ 也发现, 当煆烧温度高于 $350{ }^{\circ} \mathrm{C}$ 时, MFI 分子篮膜在脱除 模板剂时更容易产生缺陷。因此, 近年来很多研 究者致力于开发在较低温度下脱除分子笁颗粒和 分子篮膜内有机模板剂的方法, 如紫外/臭氧 法 ${ }^{21,34,35}$ 、液相氧化法 ${ }^{36-38}$ 和低温加氢裂解法 ${ }^{39,40}$ 等。 其中, 低温加氢裂解法可以在 $300{ }^{\circ} \mathrm{C}$ 以下的中低 温条件下有效去除高硅 MFI 分子篮晶体内的 TPAOH 模板剂, 是一种工艺简单且成本较低的低 温热处理脱模板剂方法。

为更好地解决上述两个分子篮膜制备领域的 关键科学问题, 本文研究了采用高孔隙率的廉价 $\alpha-\mathrm{Al}_{2} \mathrm{O}_{3}$ 片为载体合成完备分子篮膜的工艺。以适 度的晶种层作为过渡层, 省去了复杂而繁琐的载 体预处理工艺; 通过调控合成液配比, 直接在未 经处理的大孔粗䊁载体上制备出完备的高硅 MFI 分子篎膜。进而, 成功将低温加氢裂解方法应用 于高硅 MFI 分子篎膜内模板剂的脱除过程, 并提 出了一种改进的两步法低温脱除分子篮膜内有机 模板剂的工艺。

\section{2 实验部分}

\section{1 实验试剂与材料}

试剂: 正硅酸乙酯(TEOS, AR, 天津市大茂 化学试剂厂), 质量分数为 $25 \%$ 的四丙基氢氧化铵 (AR, 天津光复精细化工研究所)。实验所用载体 为自制的大孔 $\alpha-\mathrm{Al}_{2} \mathrm{O}_{3}$ 片, 厚度和直径分别为 2 和 $25 \mathrm{~mm}$, 平均孔径为 $510 \mathrm{~nm}$, 孔隙率为 $69.8 \%$ (压 永法测得)。

\section{2 高硅 MFI 分子笠膜的制备}

\section{2 .1 晶种的制备}

按照摩尔比为 $1 \mathrm{TEOS}: 0.36 \mathrm{TPAOH}: 60 \mathrm{H}_{2} \mathrm{O}$ 的 比例配制合成液, 室温下搅拌 $5 \mathrm{~h}$, 将合成液倒入 以聚四氟乙烯为内祄的不锈钢反应釜中, 密封, $150{ }^{\circ} \mathrm{C}$ 下晶化 $20 \mathrm{~h}$; 冷却后取出, 并反复经过去离 子水超声清洗、离心分离得到晶种分子篮, 置于 $105^{\circ} \mathrm{C}$ 烘箱中干燥备用; 所制备的分子篮晶种大小 均匀, 粒径约为 $250 \mathrm{~nm}$ 。

\subsection{2 晶种层的制备}

采用旋涂法在载体片表面预涂晶种 ${ }^{41}$ 。配制一 定浓度的晶种液, 并超声 $15 \mathrm{~min}$, 将载体片固定于 匀胶机上, 涂覆适量晶种液, 迅速打开旋转按 钮, 在较低转速 $\left(500 \mathrm{r} \cdot \mathrm{min}^{-1}\right)$ 下停留 $6 \mathrm{~s}$, 之后在较 高转速 $\left(2000 \mathrm{r} \cdot \mathrm{min}^{-1}\right)$ 下停留 $20 \mathrm{~s}$, 均匀涂覆晶种, 最后置于 $60^{\circ} \mathrm{C}$ 烘箱中烘干备用。

\section{2 .3 分子䇻膜的制备}

按照摩尔比为 $1 \mathrm{TEOS}: 0.2 \mathrm{TPAOH}: 1000 \mathrm{H}_{2} \mathrm{O}$ 配制分子篮合成液 $\left(\mathrm{L}_{1}\right)$ : 在剧烈摚拌的条件下, 将 TEOS 缓慢加入装有 TPAOH 及水的三口烧瓶中, 室温下搅拌老化 $5 \mathrm{~h}$ 后得到澄清的合成液。将预涂 过晶种的载体片置于反应釜中，倒入适量 $\mathrm{L}_{1}$ 合成 液, 在 $150{ }^{\circ} \mathrm{C}$ 下晶化 $8 \mathrm{~h}$ 得到一次合成膜片 $\left(\mathrm{M}_{1}\right)$ 。 将膜片取出后用去离子水冲洗, 在 $60^{\circ} \mathrm{C}$ 烘箱中干 燥。再配制摩尔比为 $1 \mathrm{TEOS}: 0.2 \mathrm{TPAOH}: 1500 \mathrm{H}_{2} \mathrm{O}$ 的分子篮合成液 $\left(\mathrm{L}_{2}\right)$, 将一次合成后膜片重新放入 反应釜中，倒入适量 $\mathrm{L}_{2}$ 合成液， $150{ }^{\circ} \mathrm{C}$ 条件下水 热合成 $8 \mathrm{~h}$ 得到二次合成膜片 $\left(\mathrm{M}_{2}\right)$, 清洗并烘干; 重复二次合成过程, 制得三次合成膜片 $\left(\mathrm{M}_{3}\right)$, 清洗 并烘干备用。最后, 对经过三次合成且验漏达标 的完备分子篮膜片 $\left(\mathrm{M}_{3}\right)$, 分别采用三种方法, 在不 同条件下进行脱除有机模板剂热处理。

\section{3 分子筛膜内有机模板剂的脱除}

\subsection{1 低温加氢裂解脱除分子篮膜内有机模板剂} 低温加氢裂解脱除分子笁膜内有机模板剂的 
实验流程详见相关文献 ${ }^{40}$ 。先将制备好的膜片 $\mathrm{M}_{3}$ 置 于气氛炉中, 然后将炉内抽至真空度为 $\sim 09$ $\mathrm{MPa}$, 再缓慢通入氢气至常压后, 以 $1^{\circ} \mathrm{C} \cdot \mathrm{min}^{-1}$ 升 温速率升至 $300{ }^{\circ} \mathrm{C}$, 恒温 $24 \mathrm{~h}$, 自然降温至室温, 取出; 在模板剂脱除过程中氢气流速为 $250 \mathrm{~mL}$. $\mathrm{min}^{-1}$ 左右。经此方法脱除模板剂后的膜片命名为 $\mathrm{M}_{3-1}$ 。

\subsection{2 低温两步法脱除分子笠膜内有机模板剂}

将上述加氢裂解法脱除模板剂后的膜片 $\mathrm{M}_{3-1}$, 再次置于气氛炉中, 通入流动空气, 以 $1^{\circ} \mathrm{C} \cdot \mathrm{min}^{-1}$ 升温速率升至 $300^{\circ} \mathrm{C}$, 恒温 $5 \mathrm{~h}$, 自然降温至室 温, 取出; 其中空气流速同样为 $250 \mathrm{~mL} \cdot \mathrm{min}^{-1}$ 左 右。经此方法脱除模板剂后的膜片命名为 $\mathrm{M}_{3-2}$ 。

\subsection{3 传统高温啭烧法脱除分子篮膜内有机模板剂}

将制备好的分子篮膜片 $\mathrm{M}_{3}$ 置于马弗炉中, 以 $0.2{ }^{\circ} \mathrm{C} \cdot \mathrm{min}^{-1}$ 的升温速率升至 $500{ }^{\circ} \mathrm{C}$, 恒温 $10 \mathrm{~h}$ 后, 同样以 $0.2{ }^{\circ} \mathrm{C} \cdot \mathrm{min}^{-1}$ 的降温速率由 $500{ }^{\circ} \mathrm{C}$ 降温 至室温, 经此方法脱除模板剂后的膜片命名为 $\mathrm{M}_{3-3}$ 。

\section{4 高硅 MFI分子篮膜的气体渗透实验}

分子笁膜的验漏与气体分离实验的操作流程 与文献 ${ }^{16,41}$ 中一致。将制备好的膜片放入不锈钢膜 组件中, 连接好膜组件气路。通过调节进出口气 体流量使得膜前端保持一定的压力, 膜后端连接 皇泡流量计测量膜片的气体渗透通量, 或连接气 相色谱(GC2014)考察膜片的气体分离效果。单组 分气体渗透实验所用气体为高纯氮气 $(99.999 \%)$; 双组分气体膜分离实验过程使用气体为 $50 \% \mathrm{CO}_{2} /$ $50 \% \mathrm{~N}_{2}$ 的混合标准气。

通过单组分气体的渗透数据可以简单估算多 孔陶瓷材料的平均孔径 ${ }^{42,43}$, 如公式(1)-(4)所示, 其中气体的渗透通量 $J$ 与平均压差 $P_{(\mathrm{av})}$ 呈线性关 系，根据这一线性关系可以计算多孔陶瓷材料的 平均孔径。虽然该公式系列多用于计算平均孔径 大于 $50 \mathrm{~nm}$ 的大孔陶瓷材料, 但也可用于判定生长 在多孔氧化铝载体上的分子笁复合膜是否存在较 大缺陷。本文结合膜片脱除模板剂后的 $\mathrm{N}_{2}$ 气体渗 透数据, 利用该系列公式来计算脱除模板剂时膜 内可能形成的较大缺陷 $(>50 \mathrm{~nm})$ 情况。

$$
\begin{aligned}
& J=Q / A\left(P_{\mathrm{h}}-P_{1}\right) \\
& J=\alpha+\beta P_{(\text {av) }} \\
& P_{(\text {av) }}=\left(P_{\mathrm{h}}+P_{1}\right) / 2 \\
& d_{\mathrm{p}}=16.964 \eta \sqrt{\frac{R T}{M}}\left(\frac{\beta}{\alpha}\right)
\end{aligned}
$$

式(1)中 $Q$ 为渗透气流量 $\left(\mathrm{mol} \cdot \mathrm{s}^{-1}\right), A$ 为膜片的有效 面积 $\left(\mathrm{m}^{2}\right), P_{\mathrm{h}}$ 为膜前端高压侧压力 $(\mathrm{Pa}), P_{1}$ 为膜后 端低压侧压力 $(\mathrm{Pa}), J$ 为一定压差下单位膜面积的 气体渗透通量 $\left(\mathrm{mol} \cdot \mathrm{m}^{-2} \cdot \mathrm{s}^{-1} \cdot \mathrm{Pa}^{-1}\right)$; 式(2)中 $P_{(\mathrm{av})}$ 为膜 片前后端的平均压力 $(\mathrm{Pa})$, 式中的 $\alpha$ 和 $\beta$ 可由渗透 通量与平均压力的线性拟合计算得到; 式(4)中 $d_{\mathrm{P}}$ 为多孔材料平均孔径 $(\mathrm{nm}), \eta$ 为氮气在此温度下的 粘度系数 $(\mathrm{Pa} \cdot \mathrm{s}), R$ 为气体常数 $\left(\mathrm{J} \cdot \mathrm{mol}^{-1} \cdot \mathrm{K}^{-1}\right), T$ 为 温度 $(\mathrm{K}), M$ 为氮气分子的摩尔质量 $\left(\mathrm{g} \cdot \mathrm{mol}^{-1}\right)$ 。

\section{5 分析仪器与表征方法}

\subsection{1 分析仪器}

本文中所使用的分析仪器包括以下几种: 日 本日立公司的 S4800场发射扫描电镜(SEM); 荷兰 PANalytical 公司的 X' Pert Pro MPD X 射线衍射仪 (XRD), 测试条件为 $\mathrm{Cu} K_{a}$ 辐射 $(\lambda=0.15406 \mathrm{~nm})$, 靶 电流 $40 \mathrm{~mA}$, 靶电压 $40 \mathrm{kV}$, 扫描范围 $5^{\circ}-50^{\circ}$; 法 国 Horiba Jobin Yvon 公司的 LabRAM HR800-LS55 型激光共聚焦拉曼光谱仪, 激光器的入射光波长 为 $325 \mathrm{~nm}$, 扫描范围为 $200-4000 \mathrm{~cm}^{-1}$; 日本岛津 公司的气相色谱仪 GC2014, 热导检测器(TCD), Propark Q 和 $5 \mathrm{~A}$ 分子篮色谱柱。

\section{5 .2 分子節膜的 XRD 表征分析}

选取 XRD 谱图中 $2 \theta=7.9^{\circ}$ 处(101)取向特征峰 强度与 $2 \theta=8.9^{\circ}$ 处 (200)取向特征峰强度的比值来 表征膜内分子篮晶体生长取向的变化规律, 计作 $I_{101} / I_{200}$; 该值越大说明分子篎膜的 $h 0 h$-轴取向越显 著。选取 XRD 谱图中 $2 \theta=7.9^{\circ}$ 处分子篎膜(101)取 向特征峰强度与 $2 \theta=25.6^{\circ}$ 处氧化铝载体特征峰强 度的比值来表征分子篮膜完备性的变化规律, 计 作 $I_{101} / I_{\alpha-\mathrm{Al}_{2} \mathrm{O}}$; 该值越大说明分子篮膜内晶体交联越 致密。

\section{3 结果与讨论}

\section{1 高硅 MFI 分子筛膜的表征}

图 1 为 $\alpha-\mathrm{Al}_{2} \mathrm{O}_{3}$ 载体片和晶种层的 SEM 照片。 从图 1(a,b) 可以看出, 由于所采用的大孔 $\alpha-\mathrm{Al}_{2} \mathrm{O}_{3}$ 载体未经打磨清洗等繁琐的表面预处理工序, 所 以载体表面极为粗糙不平; 表面颗粒大小不均, 主要颗粒粒径为 $1-10 \mu \mathrm{m}$ (图 1a), 其粗糙表面的凹 凸范围达到 2-5 $\mu \mathrm{m}$ (图 1b)。图 1(c, d) 的结果表 明, 采用旋涂分子篮晶种的工艺, 可以在粗䊁的 $\alpha-\mathrm{Al}_{2} \mathrm{O}_{3}$ 载体上覆盖一层紧密且无取向排列的分子 篮晶种层(见图 2 中 XRD 结果)。但是, 由于载体表 

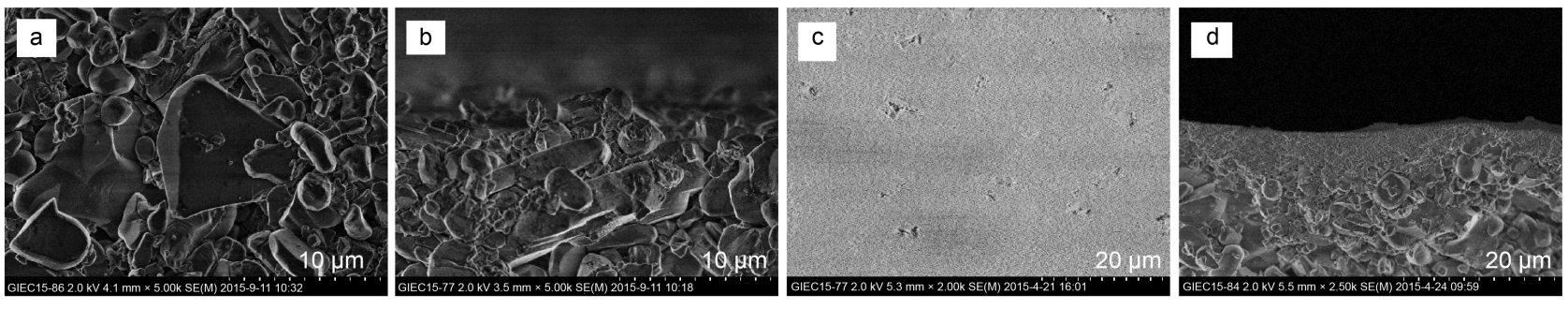

图 $1 \alpha-\mathrm{Al}_{2} \mathrm{O}_{3}$ 载体片及晶种层的 $\mathrm{SEM}$ 图片

Fig.1 $\mathrm{SEM}$ images of $\alpha-\mathrm{Al}_{2} \mathrm{O}_{3}$ supports and seeds layers

(a) top view and (b) cross section view of $\alpha-\mathrm{Al}_{2} \mathrm{O}_{3}$ supports; (c) top view and (d) cross section view of seed layers

面凹凸不平的原因, 晶种层厚度并不均匀, 约为 3-6 $\mu \mathrm{m}$ ，且仍有少许表面覆盖不完全(图 1c)。

文献 ${ }^{41,44}$ 表明, 适度降低合成液中的 $\mathrm{H}_{2} \mathrm{O} / \mathrm{Si}$ 摩 尔比有利于分子篮晶体之间的相互交联生长。因 而实验中, 第一次成膜时采用 $n\left(\mathrm{H}_{2} \mathrm{O}\right) / n(\mathrm{Si})=1000$ 的 $\mathrm{L}_{1}$ 合成液。如图 $3(\mathrm{a}, \mathrm{b})$ 所示, 在 $150{ }^{\circ} \mathrm{C}$ 下水热晶 化 $8 \mathrm{~h}$ 后, 分子篮晶体之间已经较好地交联生长, 且最上层晶体已向面外生出一层厚度约为 $1.5 \mu \mathrm{m}$ 的致密且为 $h 0 h$-轴取向的分子篮薄膜层 (图 2); 但 其 $\mathrm{N}_{2}$ 验漏通量高达 $1.1 \times 10^{-8} \mathrm{~mol} \cdot \mathrm{m}^{-2} \cdot \mathrm{s}^{-1} \cdot \mathrm{Pa}^{-1}$ (表 $1)$, 表明一次合成后的分子篎膜还存在较多晶间缺 陷, 尚需进一步交联生长。此外, 由于 $\mathrm{L}_{1}$ 合成液 的 $\mathrm{pH}$ 值较低 $(\mathrm{pH}=9-10)$, 且一次合成时间较短, 可有效抑制 $\alpha-\mathrm{Al}_{2} \mathrm{O}_{3}$ 载体中铝元素的溶出 ${ }^{41}$, 有利于 高硅 MFI 分子篮膜的制备。为了进一步抑制分子 篮晶体的面外生长, 实验中采用 $n\left(\mathrm{H}_{2} \mathrm{O}\right) / n(\mathrm{Si})=$ 1500 的 $\mathrm{L}_{2}$ 合成液配比进行分子節膜的第二次和第 三次晶化合成。如图 3 所示, 随着合成次数的增 加, 分子篮膜表面的晶体尺寸逐渐增大, 晶体间 的结合也由疏松变得致密, 膜表面也更为平整;

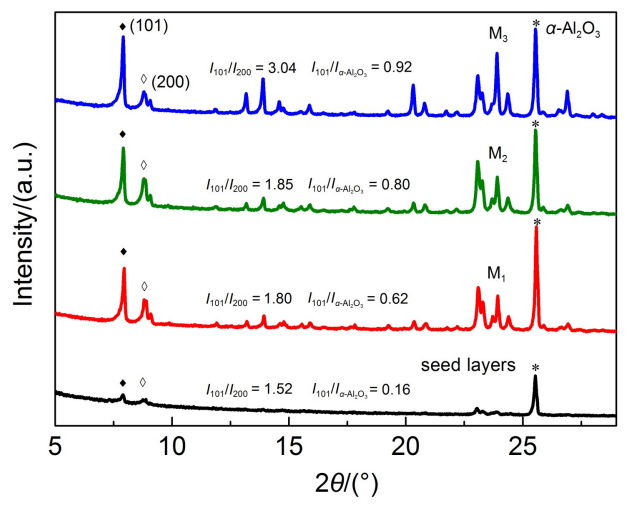

图 2 分子筛晶种层和分子篮膜的XRD 图

Fig.2 XRD patterns of the seed layers and zeolite membranes

$\mathrm{M}_{1}, \mathrm{M}_{2}$, and $\mathrm{M}_{3}$ represent the first-time, second-time, and third-time synthesized membranes, respectively.
一次合成后, 分子篮膜的总厚度不再增加, 约为 8 $\mu \mathrm{m}$ 。然而, 分子篎膜的内部结构仍然会发生一定 的变化, 其侧面结构 SEM 照片表明, 所制备的复 合分子篮膜可以分为 3 个部分, 即分子篮膜致密 层、晶种过渡层和载体层。表 1 中列出了分子篮 膜内部结构随着合成次数增加的变化规律, 即 分子篮膜的膜层总厚度保持不变, 致密层的厚 度逐渐增加, 过渡层的厚度逐渐减少。这充分 说明 $n\left(\mathrm{H}_{2} \mathrm{O}\right) / n(\mathrm{Si})=1500$ 的 $\mathrm{L}_{2}$ 合成液更有利于分子 筛晶体的面内交联生长; 因而, 在二次合成和三 次合成过程中, 分子䇻膜的总厚度没有增加, 但 $\mathrm{N}_{2}$ 验漏通量却大幅下降, 即可能存在的晶间缺陷 逐渐变少, 经过三次晶化合成后分子篎膜片的渗 透通量减小至 $7.1 \times 10^{-11} \mathrm{~mol} \cdot \mathrm{m}^{-2} \cdot \mathrm{s}^{-1} \cdot \mathrm{Pa}^{-1}$, 最终成 为较完备的分子篎膜。此外, XRD 谱图结果(图 2) 也表明所制备的分子篮膜具备了高硅 MFI 分子篮 膜的典型特征峰; 且随着合成次数的增加, 谱图 中分子篮膜(101)和(200)特征峰的峰强度比 $I_{101} / I_{200}$ 由 1.80 增加至 3.04 , 分子䇻膜 (101) 处特征峰与 $\alpha-$ $\mathrm{Al}_{2} \mathrm{O}_{3}$ 载体的特征峰的峰强度比 $I_{101} / I_{\alpha-\mathrm{Al}_{2} \mathrm{O}_{3}}$ 由 0.62 增 加至 0.96 ; 说明分子篮晶体是优先沿 $h 0 h$-轴取向交 联生长, 且分子篮膜层的致密度及厚度随合成次 数的增加而逐步增大。

\section{2 分子篮膜内有机模板剂的脱除}

为了有效避免分子篮膜在热处理脱除模板剂 过程中形成晶间缺陷或裂纹, 本实验对比考察了 三种有机模板剂脱除方法: 传统高温敾烧法、低 温加氢裂解法和低温加氢裂解耦合低温空气氧化 的低温两步法。实验选取同一批次合成的 3 个高硅 MFI 分子篮膜片 (其 $\mathrm{N}_{2}$ 验漏通量均为 $(5-7) \times 10^{-11}$ $\mathrm{mol} \cdot \mathrm{m}^{-2} \cdot \mathrm{s}^{-1} \cdot \mathrm{Pa}^{-1}$, 考察其膜内有机模板剂的脱除 效果, 以脱除模板剂后分子篎膜的拉曼谱图(图 4) 和 $\mathrm{N}_{2}$ 单组分气体渗透性能(图 5)来表征。有机模板 剂的拉曼位移特征峰包括: $1460 \mathrm{~cm}^{-1}$ 处的 $-\mathrm{CH}_{3}$ 变 

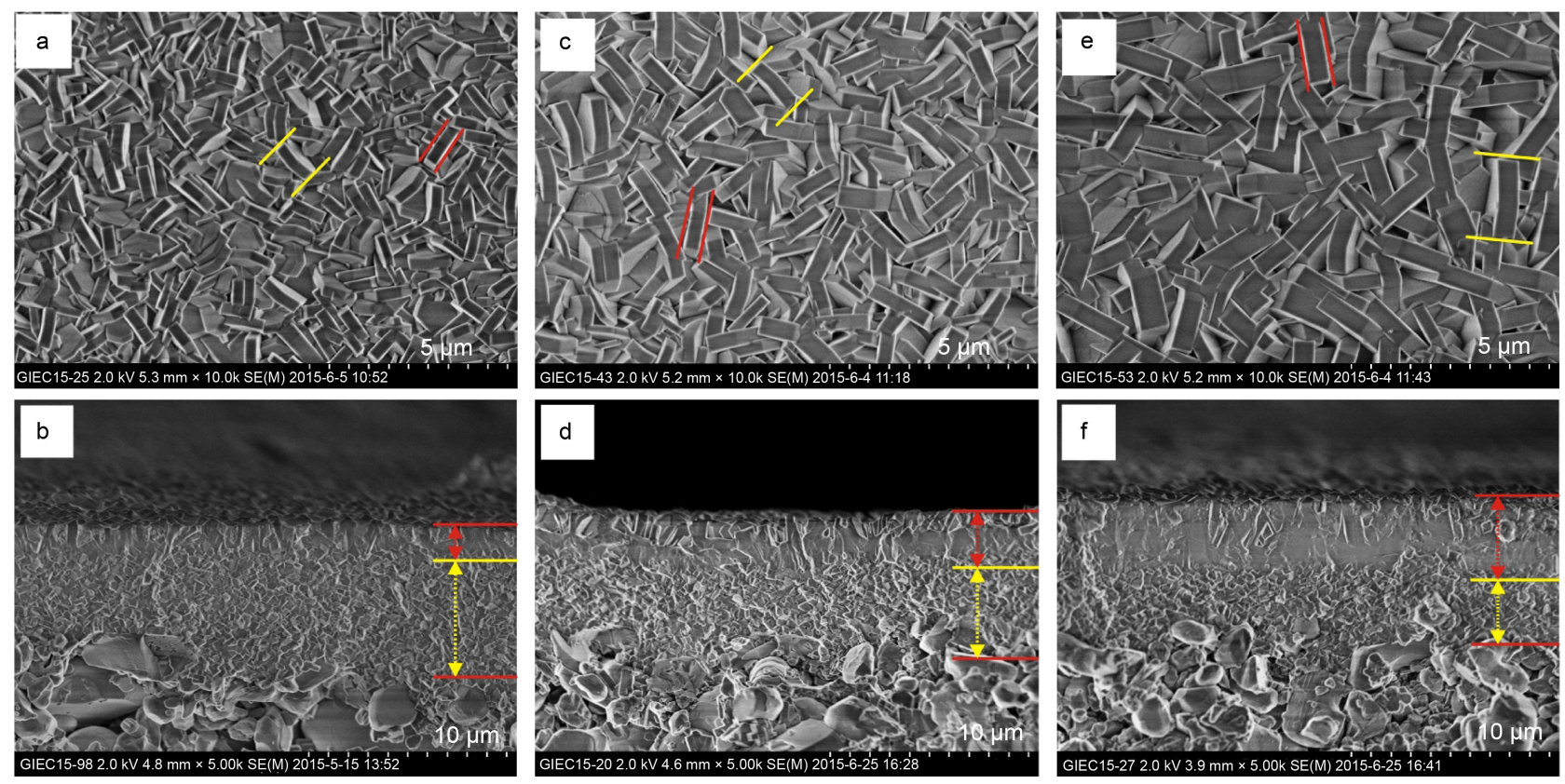

图 3 不同合成次数的分子篮膜 SEM图片

Fig.3 SEM images of zeolite membranes with different synthesis times

(a) top view and (b) cross section view of $\mathrm{M}_{1}$; (c) top view and (d) cross section view of $\mathrm{M}_{2}$; (e) top view and (f) cross section view of $\mathrm{M}_{3}$

表 1 不同合成次数的分子篮膜膜层变化量及 $\mathbf{N}_{2}$ 验漏结果

Table 1 Size parameters and $\mathrm{N}_{2}$ leaking results of zeolite membranes with different synthesis times

\begin{tabular}{ccccc}
\hline \multirow{2}{*}{ Membrane } & \multicolumn{3}{c}{ Thickness of membranes $/ \mu \mathrm{m}$} & \multirow{2}{*}{$J_{\mathrm{N}_{2}} /\left(\mathrm{mol} \cdot \mathrm{m}^{-2} \cdot \mathrm{s}^{-1} \cdot \mathrm{Pa}^{-1}\right)$} \\
\cline { 2 - 4 } & total film & dense layer & intermediate layer & $1.1 \times 10^{-8}$ \\
$\mathrm{M}_{1}$ & $\sim 8$ & $\sim 1.5$ & $\sim 6.5$ & $5.8 \times 10^{-10}$ \\
$\mathrm{M}_{2}$ & $\sim 8$ & $\sim 3.0$ & $\sim 5.0$ & $7.1 \times 10^{-11}$ \\
$\mathrm{M}_{3}$ & $\sim 8$ & $\sim 5.0$ & $\sim 3.0$ & \\
\hline
\end{tabular}

$J_{\mathrm{N}_{2}}$ represents $\mathrm{N}_{2}$ leaking test results of membranes.

形振动峰, $1600 \mathrm{~cm}^{-1}$ 处的 $\mathrm{C}-\mathrm{C}$ 伸缩振动峰以及 $2900-3000 \mathrm{~cm}^{-1}$ 处对称和反对称 $-\mathrm{CH}_{3}$ 的三个伸缩 振动峰。从图 4 中不难发现, 虽然 $300{ }^{\circ} \mathrm{C}$ 下加氢裂 解法可以有效脱除分子篮晶种颗粒 (seeds, 粒径为 $250 \mathrm{~nm}$ )中的有机模板剂, 但在 $300{ }^{\circ} \mathrm{C}$ 下加氢裂解 脱除模板剂后的分子篮膜 $\left(\mathrm{M}_{3-1}\right)$ 内仍残余少量有机 物。这说明由于分子篮膜具有致密且完备的膜层 结构, 其低温脱除有机模板剂的难度较大, 低温 加氢裂解法仅能部分脱除高硅 MFI 分子篮膜内的 有机模板剂。因此, 本实验又提出一种低温两步 法脱除工艺, 在第一步低温加氢裂解脱除模板剂 使分子笁孔道部分打开后, 第二步采用空气氧化 性气氛在 $300{ }^{\circ} \mathrm{C}$ 低温条件下进行膜内有机模板剂 的二次脱除, 目的是促进膜内有机残留物的低温 氧化反应。图 4 的拉曼结果表明, 低温两步法处理 后的膜片 $\left(\mathrm{M}_{3-2}\right)$ 和高温衫烧处理后的膜片 $\left(\mathrm{M}_{3-3}\right)$, 均 未检测到有机模板剂的特征峰。因此, 采用低温
两步法脱除工艺可以达到传统高温衫烧的脱除效

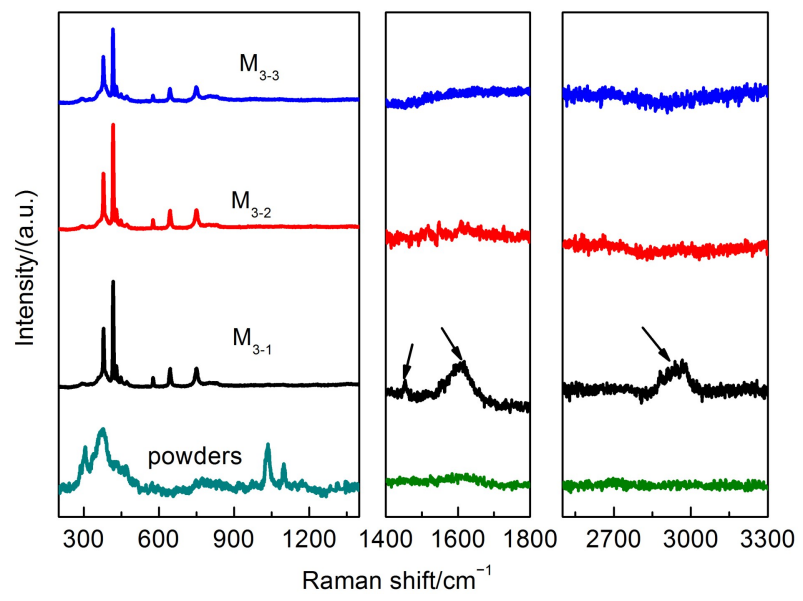

图4 脱除模板剂后分子篮膜片及分子節粉末的拉曼谱图

Fig.4 Raman spectra of zeolite membranes and zeolite powders with template removal

$\mathrm{M}_{3-1}: \mathrm{M}_{3}$ membrane detemplated by hydrocracking at $300{ }^{\circ} \mathrm{C}$;

$\mathrm{M}_{3-2}: \mathrm{M}_{3}$ membrane detemplated by two-step method at $300{ }^{\circ} \mathrm{C}$; $\mathrm{M}_{3-3}: \mathrm{M}_{3}$ membrane detemplated by calcination at $500^{\circ} \mathrm{C}$ 


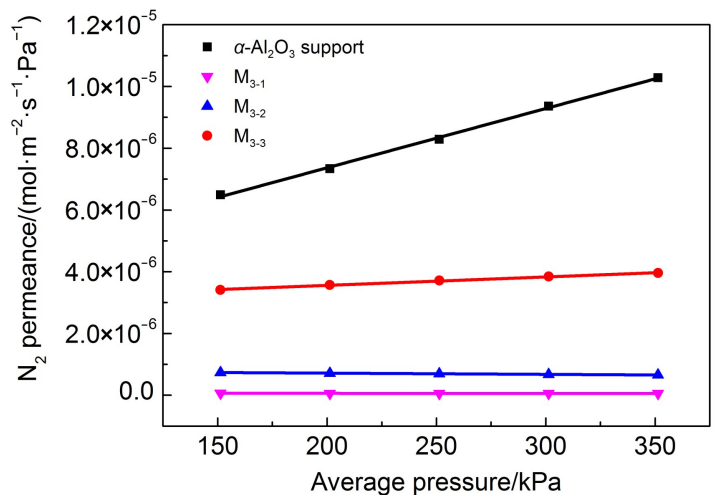

图 $5 \alpha-\mathrm{Al}_{2} \mathrm{O}_{3}$ 载体和分子篮膜的 $\mathrm{N}_{2}$ 气体渗透通量

Fig.5 $\mathrm{N}_{2}$ permeance of $\alpha-\mathrm{Al}_{2} \mathrm{O}_{3}$ supports and zeolite membranes

果, 最终得到孔道畅通的分子篮膜。

此外, 膜片 $M_{3-1} 、 M_{3-2}$ 和 $M_{3-3}$ 的 $N_{2}$ 单组分气体 渗透数据(图 5)也表明, 随着有机模板剂的脱除, 分子篮膜内孔道逐渐打开, 气体渗透通量随之增 大; 当膜内存在有机物残余时, 分子篮膜的 $\mathrm{N}_{2}$ 渗 透通量相对较低, 仅为 $(5.7-6.1) \times 10^{-8} \mathrm{~mol} \cdot \mathrm{m}^{-2}$. $\mathrm{s}^{-1} \cdot \mathrm{Pa}^{-1}$; 当充分脱除分子篮膜内有机模板剂后, 分子篮膜的 $\mathrm{N}_{2}$ 渗透通量显著增大。高温㷽烧膜片 $\mathrm{M}_{3-3}$ 的 $\mathrm{N}_{2}$ 渗透通量甚至达到了 $(3.4-3.9) \times 10^{-6} \mathrm{~mol} \cdot$ $\mathrm{m}^{-2} \cdot \mathrm{s}^{-1} \cdot \mathrm{Pa}^{-1}$, 接近于大孔氧化铝载体片的 $\mathrm{N}_{2}$ 渗透 通量。这表明高温炦烧后分子笁膜片很可能存在 较大的晶间缺陷或裂纹。根据多孔材料的 $\mathrm{N}_{2}$ 渗透 数据, 采用公式(2)和(4)计算载体和不同膜片的平 均孔径。表 2 中列出了 $\mathrm{N}_{2}$ 渗透数据的线性拟合结果 和不同膜片的计算孔径。结果表明: 大孔 $\alpha-\mathrm{Al}_{2} \mathrm{O}_{3}$ 载体的平均孔径大小为 $484 \mathrm{~nm}$, 与压录法测量结 果接近; 高温衫烧膜片 $\mathrm{M}_{3-3}$ 的平均孔径达到 83 $\mathrm{nm}$, 远大于分子篎孔道的直径。由此推测, 在高 温殷烧过程中, 分子䇻膜片容易形成较大尺寸的 晶间缺陷或裂纹, 从而会严重影响其气体分离性 能。对于低温脱除模板剂的膜片 $\mathrm{M}_{3-1}$ 和 $\mathrm{M}_{3-2}$, 其拟 合斜率 $\beta$ 为负值, 说明其平均孔径远小于公式(4)的 计算范围, 进而说明低温两步法脱除模板剂工 艺, 可以避免分子篎膜内形成较大的非晶缺陷,
有利于提高其气体分离性能。

\section{3 高硅 MFI 分子篮膜的气体分离性能}

为了进一步考察晶间缺陷对分子篮膜分离性 能的影响规律, 本文对比考察了不同条件下脱除 模板剂后分子篮膜的 $\mathrm{CO}_{2} / \mathrm{N}_{2}$ 分离效果, 结果如图 6 所示。高温热处理后膜片 $\mathrm{M}_{3-3}$ 的分离效果相对较 差, 其分离因子为 1.7-2.0, 说明在高温裧烧过程 中产生的晶间缺陷, 严重破坏了高硅 MFI 分子篮 膜的纳米孔道结构, 从而降低了其分离性能。然 而, 低温热处理的膜片 $\mathrm{M}_{3-1}$ 和 $\mathrm{M}_{3-2}$ 却均表现出较好 的 $\mathrm{CO}_{2} / \mathrm{N}_{2}$ 分离性能, 其分离因子随渗透压差的增 加而变大, 最大值均在 5.0 以上。但由于低温加氢 裂解的膜片 $\mathrm{M}_{3-1}$ 尚存有少量有机物残留, 堵塞了分 子篮的孔道, 其 $\mathrm{CO}_{2}$ 渗透通量较低, 仅为(3.6-
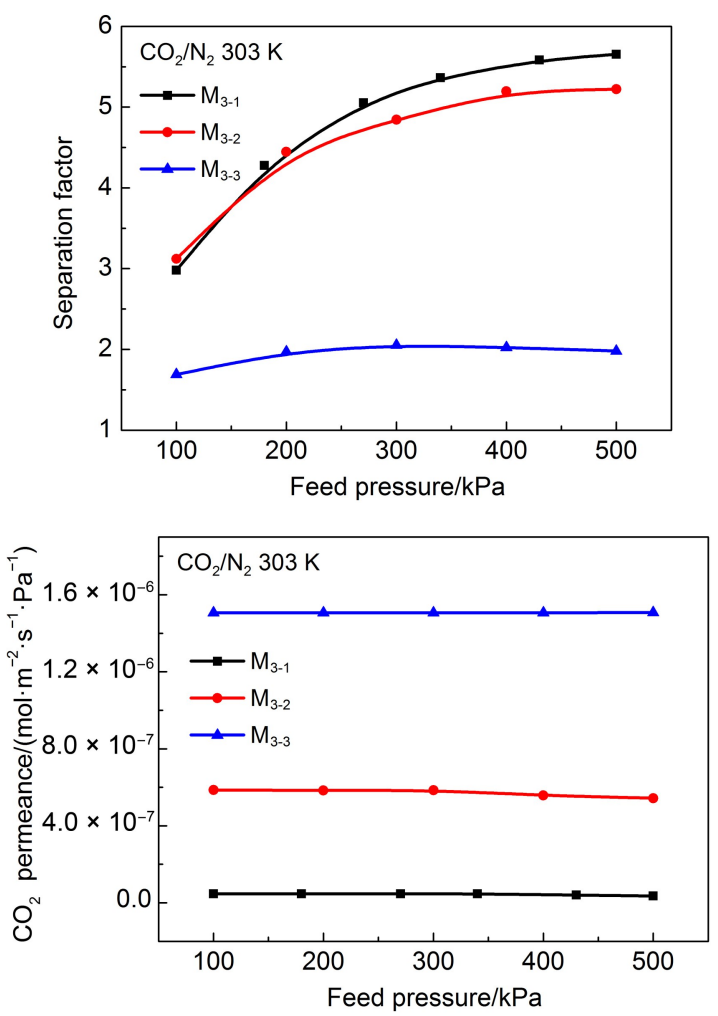

图 6 分子篮膜的 $\mathrm{CO}_{2} / \mathrm{N}_{2}$ 分离因子和 $\mathrm{CO}_{2}$ 渗透通量

Fig.6 $\mathrm{CO}_{2} / \mathrm{N}_{2}$ separation factor and $\mathrm{CO}_{2}$ permeance of zeolite membranes

表 $2 \mathbf{N}_{2}$ 渗透通量数据的线性拟合参数及膜片平均孔径

Table 2 Regression parameters and average pore sizes of the membranes from steady state $\mathrm{N}_{2}$ permeation data

\begin{tabular}{cccc}
\hline Membrane & $\alpha /\left(\mathrm{mol} \cdot \mathrm{m}^{-2} \cdot \mathrm{s}^{-1} \cdot \mathrm{Pa}^{-1}\right)^{\mathrm{a}}$ & $\beta /\left(\mathrm{mol} \cdot \mathrm{m}^{-2} \cdot \mathrm{s}^{-1} \cdot \mathrm{Pa}^{-2}\right)^{\mathrm{a}}$ & Average pore diameter $/ \mathrm{nm}^{\mathrm{b}}$ \\
\hline$\alpha-\mathrm{Al}_{2} \mathrm{O}_{3}$ supports & $3.58 \times 10^{-6}$ & $1.91 \times 10^{-11}$ & - \\
$\mathrm{M}_{3-1}$ & $6.39 \times 10^{-8}$ & $-1.81 \times 10^{-14}$ & - \\
$\mathrm{M}_{3-2}$ & $7.93 \times 10^{-7}$ & $-3.93 \times 10^{-13}$ & 83 \\
$\mathrm{M}_{3-3}$ & $3.01 \times 10^{-6}$ & $2.72 \times 10^{-12}$ & \\
\hline
\end{tabular}

${ }^{\mathrm{a}} \alpha$ and $\beta$ were given by Eq.(2); ${ }^{\mathrm{b}}$ This formula applies only to calculate the diameter of porous material which is larger than $50 \mathrm{~nm}$. 
4.6) $\times 10^{-8} \mathrm{~mol} \cdot \mathrm{m}^{-2} \cdot \mathrm{s}^{-1} \cdot \mathrm{Pa}^{-1}$; 低温两步法处理的膜 片 $\mathrm{M}_{3-2}$ 的 $\mathrm{CO}_{2}$ 渗透通量则高达 $(5.4-5.8) \times 10^{-7} \mathrm{~mol} \cdot$ $\mathrm{m}^{-2} \cdot \mathrm{s}^{-1} \cdot \mathrm{Pa}^{-1}$ 。实验结果表明, 低温两步法脱除模 板剂工艺不仅可以有效脱除高硅 MFI 分子篮膜内 的有机模板剂, 而且可以有效避免分子笁膜在模 板剂脱除过程中形成的二次缺陷。此外, 鉴于 $\mathrm{CO}_{2}$ 和 $\mathrm{N}_{2}$ 的分子动力学直径分别为 0.330 和 $0.364 \mathrm{~nm}$, 均小于 MFI 型分子篎的孔道尺寸 $(0.55 \mathrm{~nm})$, 说明 所制备的高硅 MFI 分子篎膜对 $\mathrm{CO}_{2}$ 气体具有优先吸 附分离性能, 而优先吸附在分子篮孔道内的 $\mathrm{CO}_{2}$ 分 子阻碍了 $\mathrm{N}_{2}$ 分子的扩散过程, 导致在 $\mathrm{CO}_{2} / \mathrm{N}_{2}$ 混合 体系中 $\mathrm{CO}_{2}$ 的渗透通量和选择性较高。

\section{4 结 论}

采用晶种法, 经过三次合成在未经处理的粗 糙 $\alpha-\mathrm{Al}_{2} \mathrm{O}_{3}$ 载体片上制备出包含致密膜层和过渡晶 种层的高硅 MFI 分子篮复合膜, 并通过优化合成 液中 $n\left(\mathrm{H}_{2} \mathrm{O}\right) / n(\mathrm{Si})$ 的比值, 实现了分子篮晶体的面 内 $h 0 h$-轴取向优先交联生长。提出了一种低温加 氢裂解与低温空气氧化耦合的低温两步法脱除分 子笁膜内有机模板剂的新方法。与传统高温衫烧 工艺相比, 这种新工艺不仅可以有效脱除高硅 MFI 分子篮膜内的有机模板剂, 而且可以有效避免分 子篮膜在脱除模板剂过程中形成较大的缺陷。实 验结果表明, 所制备的高硅 MFI 分子篮膜对 $\mathrm{CO}_{2}$ 气 体具有较好的优先吸附分离性能, 其对 $\mathrm{CO}_{2} / \mathrm{N}_{2}$ 二 元混合气体的 $\mathrm{CO}_{2}$ 渗透通量和选择性分离因子分别 达到 $5.6 \times 10^{-7} \mathrm{~mol} \cdot \mathrm{m}^{-2} \cdot \mathrm{s}^{-1} \cdot \mathrm{Pa}^{-1}$ 和 5.2 。但仍需指 出, 本文所报道的粗䊁载体上制备分子篮膜工艺 尚不完备，如何进一步简化合成工艺并提高 MFI 分子篮膜的 $\mathrm{CO}_{2}$ 分离性能将是今后的研究重点。

\section{References}

(1) Caro, J.; Noack, M. Microporous Mesoporous Mat. 2008, 115, 215. doi: 10.1016/j.micromeso.2008.03.008

(2) Yu, M.; Noble, R. D.; Falconer, J. L. Accounts Chem. Res. 2011, 44, 1196. doi: 10.1021/ar200083e

(3) Pina, M. P.; Mallada, R.; Arruebo, M.; Urbiztondo, M.; Navascues, N.; de la Iglesia, O.; Santamaria, J. Microporous Mesoporous Mat. 2011, 144, 19. doi: 10.1016/j. micromeso.2010.12.003

(4) Tsapatsis, M. Science 2011, 334, 767. doi: 10.1126/ science. 1205957

(5) Jiang, H. Y.; Zhang, B. Q.; Lin, Y. S.; Li, Y. D. Chin. Sci. Bull. 2004, 49, 2133. [蒋海洋, 张宝泉, 林跃生, 李永丹. 科学通报,
2004, 49, 2133.] doi: 10.1007/BF03185778

(6) Guo, Y. L.; Deng, Z. Y.; Lu, G. Z. Petrochem. Technol. 2008, 37, 865. [郭杨龙, 邓志勇, 卢冠忠. 石油化工, 2008, 37, 865.]

(7) Cheng, Y.; Li, J. S.; Liu, M.; Sun, X. Y.; Wang, L. J. China Ceram. Ind. 2004, 11(1), 40. [成 岳, 李健生, 刘 媚, 孙秀 云, 王连军. 中国陶瓷工业, 2004, 11(1), 40.]

(8) Snyder, M. A.; Tsapatsis, M. Angew. Chem. Int. Edit. 2007, 46, 7560.

(9) Cheng, Y.; Li, J. S.; Wang, L. J.; Sun, X. Y. Prog. Chem. 2006, 18, 221. [成 岳, 李健生, 王连军, 孙秀云. 化学进展, 2006, 18, 221.]

(10) Peng, Y.; Wang, Z. B. Prog. Chem. 2012, 23, 2178. [彭勇, 王 正宝. 化学进展, 2013, 23, 2178.]

(11) Lang, L.; Zhang, C.; Yin, X. L.; Wu, C. Z. Prog. Chem. 2011, 23, 1022. [郎 林, 张 超, 阴秀丽, 吴创之. 化学进展, 2011, 23, 1022.]

(12) Hedlund, J.; Sterte, J.; Anthonis, M.; Bons, A. J.; Carstensen, B.; Corcoran, N.; Cox, D.; Deckman, H.; De Gijnst, W.; de Moor, P. P.; Lai, F.; McHenry, J.; Mortier, W.; Reinoso, J. Microporous Mesoporous Mat. 2002, 52, 179. doi: 10.1016/ S1387-1811(02)00316-5

(13) Sandström, L.; Sjöberg, E.; Hedlund, J. J. Membr. Sci. 2011, 380, 232. doi: 10.1016/j.memsci.2011.07.011

(14) Zhou, H.; Korelskiy, D.; Sjöberg, E.; Hedlund, J. Microporous Mesoporous Mat. 2014, 192, 76. doi: 10.1016/j. micromeso.2013.09.017

(15) Wang, Z. X.; Yan, W. F.; Tian, D. Y.; Cao, X. J.; Yu, J. H.; Xu, R. R. Acta Phys. -Chim. Sin. 2010, 26, 2044. [王周翔, 间文付, 田大勇, 曹学静, 于吉红, 徐如人. 物理化学学报, 2010, 26, 2044.] doi: 10.3866/PKU.WHXB20100714

(16) Zhang, C.; Yan, W. F.; Yu, J. H.; Xu, R. R. Chem. J. Chin. Univ. 2012, 33, 1124. [张 聪, 间文付, 于吉红, 徐如人. 高等 学校化学学报, 2012, 33, 1124.]

(17) Zhou, M.; Zhang, B. Q.; Liu, X. F. Chin. J. Inorg. Chem. 2006, 22, 1750. [周 明, 张宝泉, 刘秀凤. 无机化学学报, 2006, 22, 1750.]

(18) Chen, H. L.; Li, Y. S.; Zhu, G. Q.; Yang, W. S. Sci. China Ser. B: Chem. 2008, 38, 777. [陈红亮, 李砚硕, 朱广奇, 杨维慎. 中 国科学(B辑: 化学), 2008, 38, 777.]

(19) Kanezashi, M.; Lin, Y. S. J. Phys. Chem. C 2009, 113, 3767. doi: 10.1021/jp804586q

(20) Tawalbeh, M.; Tezel, F. H.; Kruczek, B.; Letaief, S.; Detellier, C. J. Porous Mater. 2013, 20, 1407. doi: 10.1007/s10934-0139726-y

(21) Kosinov, N.; Auffret, C.; Sripathi, V. G. P.; Gücüyener, C.; Gascon, J.; Kapteijn, F.; Hensen, E. J. M. Microporous Mesoporous Mat. 2014, 197, 268. doi: 10.1016/j. micromeso.2014.06.022

(22) Lai, R.; Yan, Y. S.; Gavalas, G. R. Microporous Mesoporous Mat. 2000, 37, 9. doi: 10.1016/S1387-1811(99)00188-2

(23) Chen, H. L.; Li, Y. S.; Yang, W. S. J. Membr. Sci. 2007, 296, 
122. doi: 10.1016/j.memsci.2007.03.021

(24) Tuan, V. A.; Noble, R. D.; Falconer, J. L. Aiche J. 2000, 46, 1201.

(25) Wey, M. Y.; Tseng, H. H.; Chiang, C. K. J. Membr. Sci. 2013, 446, 220. doi: 10.1016/j.memsci.2013.06.051

(26) Wang, Z. B.; Yan, Y. S. Microporous Mesoporous Mat. 2001, 48, 229. doi: 10.1016/S1387-1811(01)00357-2

(27) Lee, J. S.; Lee, Y. J.; Tae, E. L.; Park, Y. S.; Yoon, K. B. Science 2003, 301, 818. doi: 10.1126/science.1086441

(28) Wang, H. B.; Dong, X. L.; Lin, Y. S. J. Membr. Sci. 2014, 450, 425. doi: 10.1016/j.memsci.2013.08.030

(29) Kim, E.; Choi, J.; Tsapatsis, M. Microporous Mesoporous Mat. 2013, 170, 1. doi: 10.1016/j.micromeso.2012.11.023

(30) Kanezashi, M.; O’Brien, J.; Lin, Y. S. Microporous Mesoporous Mat. 2007, 103, 302 doi: 10.1016/j. micromeso.2007.02.019

(31) Lang, L.; Liu, X. F.; Zhang, B. Q. Appl. Surf. Sci. 2009, 255, 4886. doi: 10.1016/j.apsusc.2008.12.030

(32) Choi, J.; Jeong, H. K.; Snyder, M. A.; Stoeger, J. A.; Masel, R. I.; Tsapatsis, M. Science 2009, 325, 590. doi: 10.1126/ science. 1176095

(33) Dong, J. H.; Lin, Y. S.; Hu, M. Z. C.; Peascoe, R. A.; Payzant, E. A. Microporous Mesoporous Mat. 2000, 34, 241. doi: 10.1016/S1387-1811(99)00175-4

(34) Li, Q. H.; Amweg, M. L.; Yee, C. K.; Navrotsky, A.; Parikh, A. N. Microporous Mesoporous Mat. 2005, 87, 45. doi: 10.1016/j. micromeso.2005.07.048
(35) Motuzas, J.; Heng, S.; Lau, P. P. S. Z.; Yeung, K. L.; Beresnevicius, Z. J.; Julbe, A. Microporous Mesoporous Mat. 2007, 99, 197. doi: 10.1016/j.micromeso.2006.06.042

(36) Melián-Cabrera, I.; Kapteijn, F.; Moulijn, J. A. Chem. Commun. 2005, NO. 21, 2744.

(37) Tago, T.; Nakasaka, Y.; Kayoda, A.; Masuda, T. Microporous Mesoporous Mat. 2008, 115, 176. doi: 10.1016/j. micromeso.2007.12.035

(38) Shen, D.; Yang, J. H.; Xiao, W.; Li, T. S.; Lu, J. M.; Wang, J. Q. Membr. Sci. Technol. 2011, 31(1), 61. [沈 东, 杨建华, 肖 伟, 李田生, 鲁金明, 王金渠. 膜科学与技术, 2011, 3l(1), 61.]

(39) Liu, X. G.; Xu, L.; Zhang, B. Q.; Liu, X. F. Microporous Mesoporous Mat. 2014, 193, 127. doi: 10.1016/j. micromeso.2013.12.034

(40) Zhao, S. H.; Lang, L.; Yin, X. L.; Yang, W. S.; Wu, C. Z. Acta Phys. -Chim. Sin. 2015, 31, 793. [ 赵淑蘅, 郎＼cjkstart林, 阴秀丽, 杨 文申, 吴创之. 物理化学学报, 2015, 31, 793.] doi: 10.3866/ PKU.WHXB201503021

(41) Lang, L.; Liu, X. F.; Zhang, B. Q. Appl. Surf. Sci. 2008, 254, 2353. doi: 10.1016/j.apsusc.2007.09.031

(42) Lin, Y. S.; Burggraaf, A. J. J. Membr. Sci. 1993, 79, 65. doi: 10.1016/0376-7388(93)85018-R

(43) Alsyouri, H. M.; Li, D.; Lin, Y. S.; Ye, Z.; Zhu, S. P. J. Membr Sci. 2006, 282, 266. doi: 10.1016/j.memsci.2006.05.046

(44) Xiao, W.; Yang, J. H.; Shen, D.; Lu, J. M.; Wang, J. Q. Microporous Mesoporous Mat. 2010, 129, 22. doi: 10.1016/j. micromeso.2009.08.036 